\title{
Transcriptional activation of the Azotobacter vinelandii polyhydroxybutyrate biosynthetic genes phbBAC by PhbR and RpoS
}

\author{
Alberto Hernandez-Eligio, Mildred Castellanos, Soledad Moreno \\ and Guadalupe Espín
}

Correspondence

Guadalupe Espín

espin@ibt.unam.mx

Received 3 June 2011

Revised 13 July 2011

Accepted 18 July 2011

\author{
Departamento de Microbiología Molecular, Instituto de Biotecnología, Universidad Nacional \\ Autónoma de México, Av. Universidad 2001, Col. Chamilpa, Cuernavaca, Morelos 62210, Mexico
}

\begin{abstract}
We previously showed that in Azotobacter vinelandii, accumulation of polyhydroxybutyrate (PHB) occurs mainly during the stationary phase, and that a mutation in phbR, encoding a transcriptional regulator of the AraC family, reduces PHB accumulation. In this study, we characterized the roles of PhbR and RpoS, a central regulator during stationary phase in bacteria, in the regulation of expression of the PHB biosynthetic operon $p h b B A C$ and $p h b R$. We showed that inactivation of rpoS reduced $\mathrm{PHB}$ accumulation, similar to the $p h b R$ mutation, and inactivation of both rpoS and $p h b R$ resulted in an inability to produce $\mathrm{PHB}$. We carried out expression studies with the wildtype, and the rpoS, phbR and double rpoS-phbR mutant strains, using quantitative RT-PCR, as well as phbB::gus $A$ and phbR:: gus $A$ gene fusions. These studies showed that both PhbR and RpoS act as activators of $p h b B$ and $p h b R$, and revealed a role for PhbR as an autoactivator. We also demonstrated that PhbR binds specifically to two almost identical 18 bp sites, TGTCACCAA $-\mathrm{N}_{4}$-CACTA and TGTCACCAA-N 4 -CAGTA, present in the $p h b B$ promoter region. The activation of $p h b B$ and $p h b R$ transcription by RpoS reported here is in agreement with the observation that accumulation of PHB in $A$. vinelandii occurs mainly during the stationary phase.
\end{abstract}

\section{INTRODUCTION}

Azotobacter vinelandii is a soil bacterium that undergoes differentiation to form cysts resistant to desiccation, and produces the polyester polyhydroxybutyrate (PHB), an industrially important polymer used as a biodegradable plastic. The sigma factor RpoS is a central regulator during the stationary phase in bacteria (for a recent review, see Navarro Llorens et al., 2010). In A. vinelandii, RpoS is involved in the formation of mature cysts (Cocotl-Yañez et al., 2011), and is also required for survival under oxidative stress and under conditions of either carbon or nitrogen starvation (Sandercock \& Page, 2008). Synthesis of $\mathrm{PHB}$ in $A$. vinelandii occurs entering the stationary phase (Martínez et al., 1997), and is carried out in three enzymic steps: a $\beta$-ketothiolase catalyses the condensation of two molecules of acetyl-CoA to produce acetoacetylCoA, which is reduced by the NADPH-dependent acetoacetyl-CoA reductase to form $\beta$-hydroxybutyrylCoA, which is polymerized by the PHB synthase. The genes encoding the $\beta$-ketothiolase $p h b A$, acetocetyl-CoA reductase $p h b B$ and PHB synthase $p h b C$ are organized in the phbBAC operon (Segura et al., 2003). The phbR gene,

Abbreviations: EMSA, electrophoretic mobility shift assay; MBP, maltose-binding protein; PHB, polyhydroxybutyrate; qRT-PCR, quantitative RT-PCR. located upstream and in the opposite direction to $p h b B$, encodes a transcriptional activator of the AraC family, and a mutation in $p h b R$ reduces accumulation of PHB (PeraltaGil et al., 2002). Transcription of the phbBAC gene cluster has been shown to start from two overlapping promoters, $\mathrm{p}_{\mathrm{B}} 1$ and $\mathrm{p}_{\mathrm{B}} 2$, and a reduction in the transcription of $p h b B$ from its $p_{\mathrm{B}} 1$ promoter in the $p h b R$ mutant has been revealed in an S1-mapping experiment (Peralta-Gil et al., 2002). A consensus sequence recognized by RpoS is present in $\mathrm{p}_{\mathrm{B}} 2$ (Peralta-Gil et al., 2002). Two start sites for the transcription of $p h b R$ have also been identified, defining the $\mathrm{p}_{\mathrm{R}} 1$ and $\mathrm{p}_{\mathrm{R}} 2$ promoters. The binding targets for some regulators of the AraC family, such as MelR from Escherichia coli, consist of repeated sequences $18 \mathrm{nt}$ long (Belyaeva et al., 2000). Two almost identical $18 \mathrm{bp}$ sequences present in the $p h b B$ regulatory region, designated R1 and R2, have been identified. Site R1 overlaps the -35 region of $\mathrm{p}_{\mathrm{B}} 1$, and $\mathrm{R} 2$ is located $3 \mathrm{nt}$ upstream of R1. Thus, $\mathrm{R} 1$ and R2 have been proposed to be PhbR-binding sites. Four other less conserved 18 bp sequences, designated R3, R4, R5 and R6, have also been identified within the intergenic $p h b R-p h b B$ region (Peralta-Gil et al., 2002).

In this study we show that synthesis of PHB, as well as transcription of $p h b B$ and $p h b R$, is abrogated in the absence of both PhbR and RpoS, and that the PhbR protein binds specifically to the R1 and R2 sequences present in the $p h b B$ 
promoter region. We also demonstrate that RpoS positively controls transcription of $p h b B$ and $p h b R$.

\section{METHODS}

Microbiological procedures. Bacterial strains and plasmids used are listed in Table 1. Medium and growth conditions were as follows. A. vinelandii was grown at $30{ }^{\circ} \mathrm{C}$ in peptone yeast medium (PY), or Burk's nitrogen-free salts medium (BS) (Kennedy et al., 1986) supplemented with $2 \%$ sucrose. E. coli strains DH5 $\alpha$ and BL21 were grown on Luria-Bertani medium (LB) at $37^{\circ} \mathrm{C}$. Antibiotic concentrations used (in $\mu \mathrm{g} \mathrm{ml}^{-1}$ ) for $A$. vinelandii and $E$. coli, respectively, were as follows: tetracycline, 20 and 20; kanamycin $(\mathrm{Km}), 5$ and 30; ampicillin (Ap), not used and 100; nalidixic acid (Nal), 20 and 20; streptomycin (Sm), 2 and 20; and gentamicin $(\mathrm{Gm}), 1.5$ and 10. A. vinelandii transformation was carried out as described by Page \& von Tigerstrom (1978); Bali et al. (1992). PHB production was measured as described previously (Segura \& Espín, 1998).

Construction of $\boldsymbol{A}$. vinelandii mutants. Primers phbRupper and phbRlower (Table 1) were used to amplify a PCR fragment containing the complete $p h b R$ gene using chromosomal DNA of strain UW136 as template. This fragment was digested with $N d e I$ and $X h o I$ restriction enzymes and cloned into the pTYB1 plasmid (NEB). The resultant plasmid, pMC1, was digested with $\mathrm{NcoI}$ (a single $\mathrm{NcoI}$ restriction site is present $375 \mathrm{bp}$ downstream from the ATG start codon). The ends of the Ncol-linearized pMC1 were made blunt by treatment with Klenow fragment (Fermentas) and used for cloning an SmaI fragment containing the Km-resistance cassette from vector pBSL97. The resultant plasmid, pMC2, was linearized with the NdeI restriction enzyme and transformed into the UW136 and JGW-S strains, generating the $p h b R$ mutant AHW1 and the rpoS-phbR double mutant AHW2, respectively. The presence of the phbR::Km mutation in these strains was confirmed by PCR (data not shown).

The $\operatorname{scr} X$ gene encodes a protein with sequence identity to $\alpha$ glucosidases. Loss of $s c r X$, either by replacement or by interruption using an antibiotic-resistance gene, did not affect the capacity of $A$. vinelandii to grow using sucrose as the sole carbon source, indicating that the $s c r X$ gene product is not essential for sucrose catabolism (Johnson et al., 2006). Therefore, the $s c r X$ gene was used as a neutral region for integration of the $p h b R-g u s A$ gene fusion. Primers scrX-F and scrX-R (Table 1) were used to amplify a $2956 \mathrm{bp}$ fragment containing the $s c r X$ gene. This fragment was cloned into pJET1.2 to give plasmid pAHX. Primers phbRint and B2 (Table 1) were used to amplify a $3.2 \mathrm{~kb}$ fragment containing the phbR::gusA-Gm gene fusion from strain JGW-R (Peralta-Gil et al., 2002). This fragment was cloned within the EcoRI site (made blunt with Klenow fragment) present in the $s c r X$ gene in plasmid pAHX. The resultant plasmid, pMC4, was linearized with XhoI and transformed into the UW136, AHW1, JGW-S and AHW2 strains. Transformants of each strain carrying phbR-gusA-Gm within the scrX gene were isolated and named AHW5, AHW6, AHW7 and AHW8, respectively. The presence of the $p h b R-g u s A-G m$ fusion within $s c r X$ was confirmed by PCR analysis (data not shown).

Primers Rfin and phbBa12 (Table 1) were used to amplify a 966 bp fragment containing the $p h b B$ gene from UW136. This fragment was cloned into plasmid pJET1.2, producing plasmid pAHB. A 2.8 Xho I fragment, carrying the $g u s A-G m$ cassette, was isolated from plasmid pSM-Gus-Gm, made blunt with the Klenow fragment, and cloned (in the same orientation as the $p h b B$ gene) into the unique SmaI site present within the $p h b B$ gene of pAHB to produce plasmid pAHB-A, carrying the $p h b B:: g u s A-G m$ fusion. Plasmid pAHB-A was transformed into the UW136, AHW1, JGW-S and AHW2 strains. Transformants of each strain carrying the $p h b B:: g u s A-G m$ fusion were isolated and named AHW9, AHW10, AHW11 and AHW12, respectively. The presence of the $p h b B:: g u s A$ gene fusion was confirmed by PCR (data not shown).

Complementation of the phbR and rpos mutants. Plasmids pAHR-KM and pAHS-GM, carrying wild-type copies of the $p h b R$ and rpoS genes, respectively, were constructed as follows. A $1902 \mathrm{bp}$ fragment containing the $p h b R$ gene was amplified from UW136 using primers bmut 2 and compphbRRev, and cloned into plasmid pJET1.2 to produce plasmid pAHR. A Km cassette from plasmid pBSL97 was inserted into the XbaI site of pAHR. The resultant plasmid, pAHR$\mathrm{KM}$, unable to replicate in $A$. vinelandii, was transformed into strain JGW-R. A transformant resistant to $\mathrm{Gm}$ and $\mathrm{Km}$, having plasmid pAHR-KM integrated into the chromosome, was isolated and named ASW3. PCR analysis showed that co-integration of pAHR-GM in ASW3 occurred between the phbR promoter and the Gm-resistance cassette (see Fig. 2b), thus allowing the wild-type $p h b R$ gene to be transcribed from its own promoter.

Primers comprpoSFw and comprpoSRev and chromosomal DNA from strain UW136 were used to amplify a $2549 \mathrm{bp}$ fragment carrying the $r p o S$ gene, which was cloned into pEJT1.2, to produce plasmid pAHS. The Gm cassette from plasmid pBSL98 was cloned into the $\mathrm{Xba \textrm {I }}$ site of pAHS to produce plasmid pAHS-GM, which was introduced into strain JGW-S by transformation to produce strain AHW4, carrying pAHS-GM integrated between the rpoS promoter and the Sp cassette (see Fig. 2c).

Nucleic acid procedures. DNA isolation and cloning, and random primer procedures were carried out as described by Sambrook et al. (1989). DNA sequencing was done with a Perkin Elmer/Applied Biosystems DNA sequencer. The sequences of all the primers used in this work are shown in Table 1.

Quantitative RT-PCR (qRT-PCR). Expression of $p h b B, p h b R$ and $r p o S$ was measured by qRT-PCR, as previously reported (Noguez et al., 2008). For RNA extraction, the cultures were grown in Burk's liquid medium supplemented with $2 \%$ sucrose as the carbon source. Cells were collected at the logarithmic or stationary phase of growth. The primers used for the qRT-PCR assays (Table 1) were as follows: phbBFw/phbBRev for $p h b B$; phbRFw/phbRRev for $p h b R$; RTuprpoS/ RTdownrpoS for $r p o S$; and gyrAFw/gyrARev for $g y r A$, which was used as internal control to normalize the results. All assays were performed in triplicate. The data are presented as fold changes of mRNA levels of the mutant strain relative to those of the wild-type.

Purification of maltose-binding protein (MBP)-PhbR. To express and purify PhbR, a 1047 bp fragment corresponding to the phbR gene was amplified by PCR using Taq Platinum High Fidelity Polymerase (Invitrogen), and phbRmalFw and phbRmalLw primers. The resultant PCR product was digested with HindIII and EcoRI, and ligated into plasmid pMAL-C2X (NEB). The ligation mix was transformed into $E$. coli $\mathrm{DH} 5 \alpha$, and a transformant carrying plasmid pMC3 was isolated. This plasmid was sequenced to confirm the presence of an intact $p h b R$ gene and transformed into E. coli BL21 (Invitrogen). Expression of the MBP-PhbR in strain BL21/pMC3 was induced by the addition of IPTG $(0.3 \mathrm{mM})$. After $2 \mathrm{~h}$ induction, protein purification was performed at $4{ }^{\circ} \mathrm{C}$ under non-denaturing conditions using an amylose resin column, as described in the manufacturer's protocol (NEB). The MBP-PhbR protein was maintained in storage buffer (10 mM Tris/ $\mathrm{HCl}, 50 \mathrm{mM}$ potassium chloride, $1 \mathrm{mM}$ DTT, $0.5 \mathrm{mM}$ EDTA, $\mathrm{pH}$ 8.0). The protein was concentrated utilizing Microcon YM-10 centrifugal filters (Amicon) and stored at $4{ }^{\circ} \mathrm{C}$. Protein concentrations were determined by the Lowry method (Lowry et al., 1951), using BSA as a standard. SDS-PAGE of the purified protein revealed the expected molecular mass of approximately $45 \mathrm{kDa}$ (data not shown). 
Table 1. Bacterial strains, plasmids and oligonucleotides used in this study

\begin{tabular}{|c|c|c|}
\hline Strain, plasmid or oligonucleotide & Description or sequence $\left(5^{\prime}\right.$ to $\left.3^{\prime}\right)$ & Source or reference \\
\hline \multicolumn{3}{|l|}{ A. vinelandii strains } \\
\hline UW136 & Wild-type & Laboratory collection \\
\hline JGW-S & UW136 with an rpoS::Sp mutation & Peralta-Gil et al. (2002) \\
\hline JGW-R & UW136 with a phbR:: Gm mutation & Peralta-Gil et al. (2002) \\
\hline AHW1 & UW136 with a phbR:: Km mutation & This study \\
\hline AHW2 & JGW-S with a $p h b R:: \mathrm{Km}$ mutation & This study \\
\hline ASW3 & JGW-R with pAHR-KM co-integrated & This study \\
\hline AHW4 & AHW2 with pAHR-GM co-integrated & This study \\
\hline AHW5 & UW136 with a phbR::gusA fusion & This study \\
\hline AHW6 & AHW1 with a $p h b R:: g u s A$ fusion & This study \\
\hline AHW7 & JGW-S with a $p h b R:: g u s A$ fusion & This study \\
\hline AHW8 & AHW2 with a $p h b R:: g u s A$ fusion & This study \\
\hline AHW9 & UW136 with a $p h b B:: g u s A$ fusion & This study \\
\hline AHW10 & AHW1 with a $p h b B:: g u s A$ fusion & This study \\
\hline AHW11 & JGW-S with a $p h b B:: g u s A$ fusion & This study \\
\hline AHW12 & AHW2 with a $p h b B:: g u s A$ fusion & This study \\
\hline \multicolumn{3}{|l|}{ E. coli strains } \\
\hline $\mathrm{DH} 5 \alpha$ & supE44 $\Delta$ lacU169 hsdR17 recA1 endA1 gyrA96 thi-1 relA1 & Hanahan (1983) \\
\hline Bl21 BL21(DE3) & $\mathrm{F}^{-} o m p T h s d S_{B}\left(\mathrm{r}_{\mathrm{B}}^{-} \mathrm{m}_{\mathrm{B}}^{-}\right) \mathrm{gal} \mathrm{dcm}$ & Invitrogen \\
\hline \multicolumn{3}{|l|}{ Plasmids } \\
\hline pJET1.2 & Cloning vector & Fermentas \\
\hline pMAL-C2 & Expression vector & NEB \\
\hline pTYB1 & Cloning vector & NEB \\
\hline pBSL97 & Plasmid with a $\mathrm{Km}$ cassette & Alexeyev et al. (1995) \\
\hline pBSL98 & Plasmid with a Gm cassette & Alexeyev et al. (1995) \\
\hline pSM-Gus-Gm & Plasmid with a gusA:: Gm cassette & Peralta-Gil et al. (2002) \\
\hline pTC2P & Plasmid with $p h b R-p h b B$ intergenic region & Peralta-Gil et al. (2002) \\
\hline pMC1 & pYTB1 derivative with $p h b R$ & This study \\
\hline pMC2 & pMC1 derivative with phbR:: Km mutation & This study \\
\hline pMC3 & pMAL-C2X derivative with $p h b R-$ male fusion & This study \\
\hline pMC4 & pAHX derivative with $p h b R:: g u s A:: \mathrm{Gm}$ & This study \\
\hline pAHX & pJET1.2 derivative with $s c r X$ & This study \\
\hline pAHB & pJET1.2 derivative with $p h b B$ & This study \\
\hline pAHB-A & pAHB derivative with $p h b B:: g u s A:: \mathrm{Gm}$ & This study \\
\hline pAHR & pJET1.2 derivative with the $p h b R$ locus & This study \\
\hline pAHS & pJET1.2 derivative with the rpoS locus & This study \\
\hline pAHR-KM & pAHR derivative with a $\mathrm{Km}$ cassette & This study \\
\hline pAHS-GM & pAHS derivative with a Gm cassette & This study \\
\hline \multicolumn{3}{|l|}{ Oligonucleotides } \\
\hline gyrAFw & CCAGCAAGGGCAAGGTCTA & Noguez et al. (2008) \\
\hline gyrARev & TCGTCCAGCGGCAACAGGT & Noguez et al. (2008) \\
\hline phbRFw & CCACATGCGTTTGGTTTCTTG & Noguez et al. (2008) \\
\hline phbRRev & TCTGCCCTAAGCGTCTCCTC & Noguez et al. (2008) \\
\hline phbBFw & TCCGCAATCCAAACGCAAAG & Noguez et al. (2008) \\
\hline phbBRev & GTGCTCTCCCAATCGGTTAC & Noguez et al. (2008) \\
\hline RTuprpos & AGGATGTCCTGGACGATGAG & Cocotl-Yañez et al. (2011) \\
\hline RTdownrpos & TCCAGCGCCCTAGTGTAGTC & Cocotl-Yañez et al. (2011) \\
\hline emsaPsup & CGAGCCGTCAGTTTGTGC & Cocotl-Yañez et al. (2011) \\
\hline psrAprim & CGAGGTCTCGGCGAATCCC & Cocotl-Yañez et al. (2011) \\
\hline phbRmalFw & TAGCGAATTCATGTTTACAGTCAGT & This study \\
\hline phbRmalLw & GATCAAGCTTTCATCCGTATGCGAC & This study \\
\hline scrX-F & GTGGTGCAACGACTCTGC & This study \\
\hline scrX-R & TCGCGGACCGTGCCTATG & This study \\
\hline Rini & CAAATCCCGCCTCGCCAAGC & This study \\
\hline Rmed & CATCGCTCTCGGCATTCTCC & This study \\
\hline Rfin & CCGATAGCAAAAAGCCACTG & This study \\
\hline
\end{tabular}


Table 1. cont.

\begin{tabular}{|lll|}
\hline Strain, plasmid or oligonucleotide & \multicolumn{1}{c|}{ Description or sequence $\mathbf{5}^{\prime}$ to $\left.\mathbf{3}^{\prime}\right)$} & Source or reference \\
\hline B1 & GGCTCATTCTCGATTGGGTC & This study \\
B2 & GTGGCTTTTTGCTATCGGGC & This study \\
B3 & GGATGGAGAATGCCGAGAGC & This study \\
bmut2 & CTTCCTTTTTTGTCGGAG & This study \\
compphbRRev & TCTCCTGCATAGTGACGG & This study \\
comprpoSFw & CAATTTCTCAGTATGGGC & This study \\
phbRint & TAGGCATGCAGGGAGTGG & This study \\
phbRupper & CTTGAGCCCTATATCCGGATT & This study \\
phbRlower & GGCTCATATGTTTACAGTCAGT & This study \\
phbBal2 & CCAACTCGAGTCCGTATGCGAC & This study \\
footphbB4 & CTAGCAGCTTTCCGAATG & This study \\
\hline
\end{tabular}

DNA gel mobility shift assays. DNA fragments corresponding to the intergenic $p h b R-p h b B$ region were amplified from $A$. vinelandii UW136 chromosomal DNA by PCR using primers Rini, Rmed, Rfin, $\mathrm{B} 1, \mathrm{~B} 2$ and B3. DNA-binding reactions were carried out in a total volume of $20 \mu \mathrm{l}$. The reactions contained DNA-binding buffer [10 mM Tris/ $\mathrm{HCl}, 50 \mathrm{mM} \mathrm{KCl}, 1 \mathrm{mM}$ DTT, $0.5 \mathrm{mM}$ EDTA, $5 \%$ (v/v) glycerol, $10 \mu \mathrm{g} \mathrm{BSA} \mu^{-1}$ ], $0.1 \mathrm{nmol}$ of each DNA fragment labelled with $\left(\gamma_{-}{ }^{32} \mathrm{P}\right) \delta \mathrm{ATP}$, and variable amounts of PhbR $(0-1 \mu \mathrm{M})$. As a negative control, a $263 \mathrm{bp}$ fragment of DNA corresponding to the promoter region of psrA was used (Cocotl-Yañez et al., 2011). This fragment was obtained by PCR using primers emsaPsup and psrAprm. DNA-binding reactions were carried out at room temperature for $25 \mathrm{~min}$, and the samples were subjected to native PAGE in $6 \%$ polyacrylamide gels in a buffer containing $90 \mathrm{mM}$ Tris, $90 \mathrm{mM} \mathrm{H} \mathrm{BO}_{3}$ and $2 \mathrm{mM}$ EDTA. The gel was dried, and radioactive signals were detected by autoradiography.

DNase I footprinting assays. Plasmid pTC2P was used as a template for PCR amplification of the $p h b B 5^{\prime}$ regulatory region. A ${ }^{32} \mathrm{P}$-labelled oligonucleotide complementary to the $5^{\prime}$ coding region of $p h b B$ (footphbR4), and an oligonucleotide complementary to the -109 to $+78 \mathrm{bp}$ region relative to the transcriptional start $\mathrm{p}_{\mathrm{B}} 1$ (Rfin), were used. The binding of MBP-PhbR to the labelled DNA fragment was performed at room temperature in buffer containing $10 \mathrm{mM}$ Tris/HCl, $50 \mathrm{mM} \mathrm{KCl,} 0.5 \mathrm{mM}$ EDTA, $1 \mathrm{mM}$ DTT, $5 \%$ (v/v) glycerol and $10 \mathrm{mg} \mathrm{BSA}^{-1}$. The reaction mixture was treated with $0.02 \mathrm{U}$ DNase I (Fermentas) for $2 \mathrm{~min}$. The DNase footprinting with MBP-PhbR was performed at $30{ }^{\circ} \mathrm{C}$. The DNA segments were separated by electrophoresis in $7 \%$ polyacrylamide/ $8 \mathrm{M}$ urea gels alongside sequencing ladders.

\section{RESULTS}

\section{Transcription of phbB and $p h b R$ in exponential and stationary phase cultures}

In A. vinelandii, the levels of the RpoS protein and of the PHB polymer significantly increase during the stationary phase (Sandercock \& Page, 2008; Martínez et al., 1997). We determined the level of rpoS mRNA by qRT-PCR in exponential and stationary cells of strain UW136 grown on PY medium. As shown in Fig. 1, the level of the rpoS transcript was about 35 -fold higher in stationary phase cells than in exponentially growing cells. Thus, it was expected that in the UW136 strain, the levels of the $p h b B$ and/or $p h b R$ mRNAs would be higher in stationary phase cells than in exponentially growing cells. Indeed, the levels of $p h b R$ and $p h b B$ were four- and eightfold higher, respectively, in stationary phase cells, as determined by qRT-PCR (Fig. 1). These data are in agreement with a role for RpoS as a positive regulator of $p h b B$ and $p h b R$ transcription.

\section{PHB accumulation and $p h b B$ transcription in the phbR and rpos mutants}

Inactivation of either $p h b R$ or $r p o S$ in strain UW136 reduced accumulation of PHB (Table 2, Fig. 2a). We carried out complementation of the $p h b R$ and rpoS mutant strains.

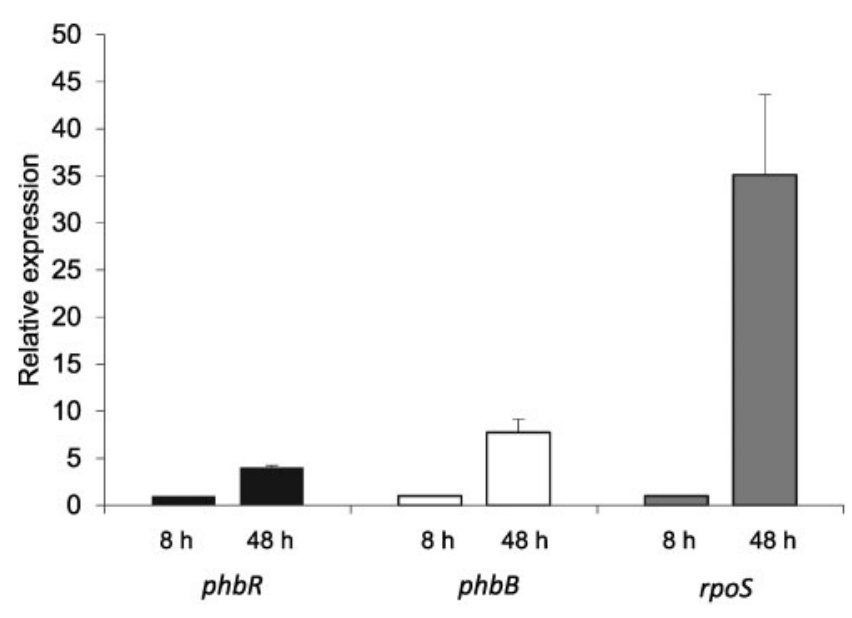

Fig. 1. Relative mRNA expression for $p h b R$, phbB and $r p o S$ in $A$. vinelandii strain UW136. qRT-PCR analysis of the expression of phbR (black bars), phbB (white bars) and rpoS (grey bars) in exponential $(8 \mathrm{~h})$ and stationary phase cells $(48 \mathrm{~h})$. The value of the mRNA level of each gene was normalized according to the level of gyrA mRNA. The data represent the means of triplicates; error bars, SD. The data are presented as the fold change of mRNA levels of stationary phase cells relative to exponentially growing cells. 
Table 2. PHB production

PHB was determined in cells grown on PY medium for $72 \mathrm{~h}$.
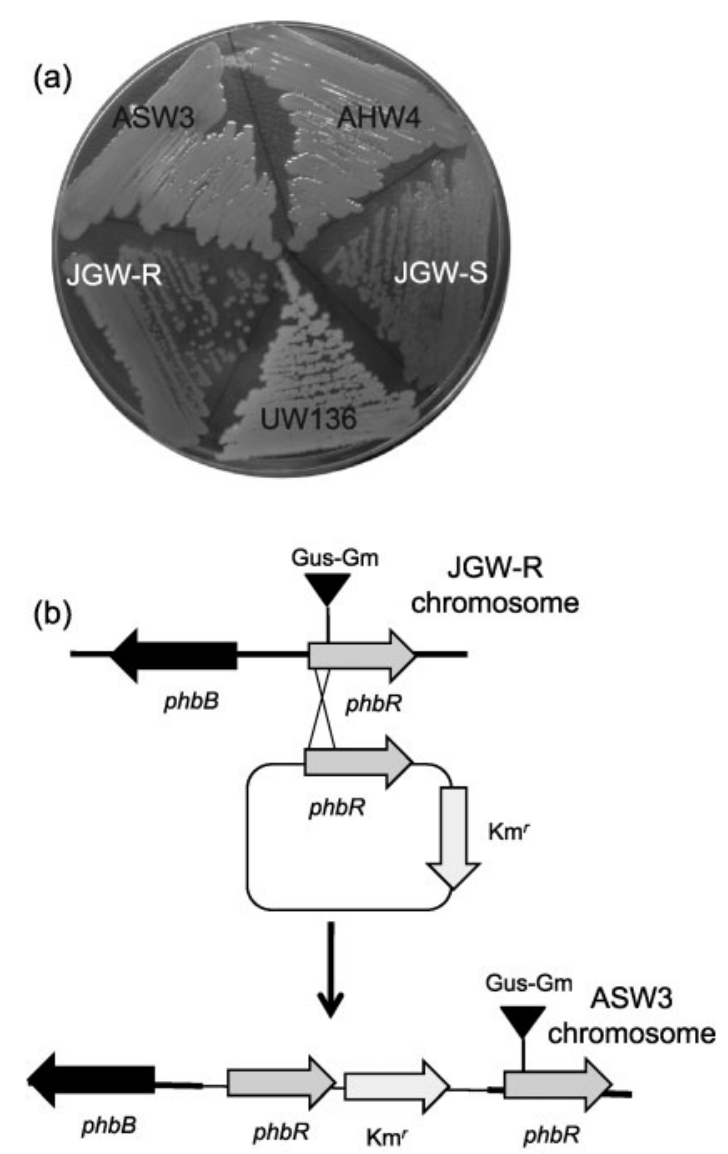

(c)

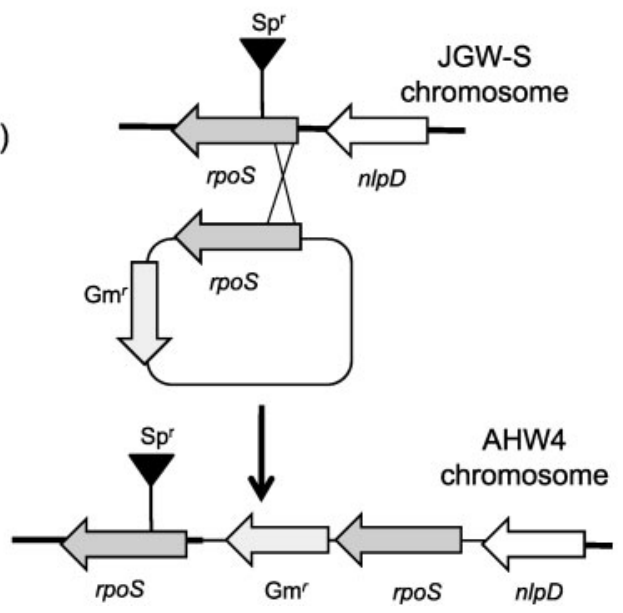

Fig. 2. Complementation of JGW-R and JGW-S mutant strains by co-integration of plasmids $\mathrm{PAHR}-\mathrm{KM}$ and $\mathrm{pAHS}-\mathrm{GM}$ carrying the phbR and rpoS genes, respectively. (a) Opacity phenotype (PHB accumulation) of UW136, JGW-R and JGW-S, and the corresponding complemented strains ASW3 and AHW4. (b, c) Schematic representations of the integration of plasmid pAHR$\mathrm{KM}$ into the chromosome of strain JGW-R (b) and pAHS-GM into the chromosome of strain JGW-S (c).

\begin{tabular}{|llc|}
\hline Strain & \multicolumn{1}{|c|}{ Genotype } & $\begin{array}{c}\text { PHB content [mg } \\
\text { (mg protein) })^{-1}\end{array}$ \\
\hline UW136 & Wild-type & $1.550 \pm 0.220$ \\
JGW-R & $p h b R:: g u s A-G m$ & $0.144 \pm 0.070$ \\
JGW-S & $r p o S:: S p$ & $0.167 \pm 0.010$ \\
AHW2 & $r p o S:: S p / p h b R:: g u s A-G m$ & $0.004 \pm 0.002$ \\
ASW3 & $p h b R:: g u s A-G m / p h b R+$ & $2.490 \pm 0.174$ \\
AHW4 & $r p o S:: S p / r p o S+$ & $2.510 \pm 0.273$ \\
& & \\
\hline
\end{tabular}

Plasmids pAHR-KM and pAHS-GM, carrying wild-type copies of the $p h b R$ and $r p o S$ genes and unable to replicate in A. vinelandii, were transformed into the JGW-R and JGW-S strains, respectively, for integration into the chromosome. Transformants ASW3 and AHW4 were isolated, and they showed the wild-type PHB accumulation phenotype (Fig. 2, Table 2), confirming that the PHB-defective phenotype was due to the $p h b R$ and rpoS mutations.

To further study the roles of RpoS and PhbR in PHB synthesis, we constructed strain AHW2, an rpoS-phbR double mutant. As expected, PHB synthesis was abrogated in the AHW2 strain (Table 2), indicating that together, RpoS and PhbR are essential for PHB synthesis. The level of $p h b B$ mRNA in strains $p h b R, r p o S$ and the double mutant $r p o S-p h b R$ was also determined by qRT-PCR. Fig. 3 (a) shows that in the absence of either RpoS or PhbR, the levels of the $p h b B$ transcripts were significantly reduced in stationary cultures. No $p h b B$ mRNA was detected in the rpoS-phbR double mutant.

The effect of the rpoS and $p h b R$ mutations on $p h b B$ expression was also analysed using a $p h b B-g u s A$ gene fusion. The induction kinetics of $p h b B$ transcription were determined in vivo by measuring the $\beta$-glucuronidase activity in strain AHW9, a UW136 derivative carrying the $p h b B-g u s A$ fusion, and their derivatives AHW10, AHW11 and AWW12, carrying $p h b R, r p o S$ and $r p o S$ - $p h b R$ mutations respectively. The $\beta$-glucuronidase activity was determined during growth on PY sucrose medium, as shown in Fig. 3(b). Growth curves were very similar in all strains carrying the $p h b B$-gus $A$ gene fusion. As shown in Fig. 3(c), $\beta$-glucuronidase activity in strain AHW9 increased upon entrance into the stationary phase, in agreement with a role for RpoS as a regulator of phbB transcription. Fig. 3(c) also shows that the rpoS, phbR and $r p o S-p h b R$ mutations significantly reduced the $\beta$ glucuronidase activity, in agreement with the reduction in the levels of $p h b B$ mRNA observed in the $p h b R, r p o S$ and $r p o S-p h b R$ mutant strains.

\section{Transcription of phbR}

The level of $p h b R$ mRNA was determined by qRT-PCR in the rpoS mutant. As shown in Fig. 4(a), the rpoS mutation reduced the concentration of the phbR transcript by $95 \%$, 
(a)
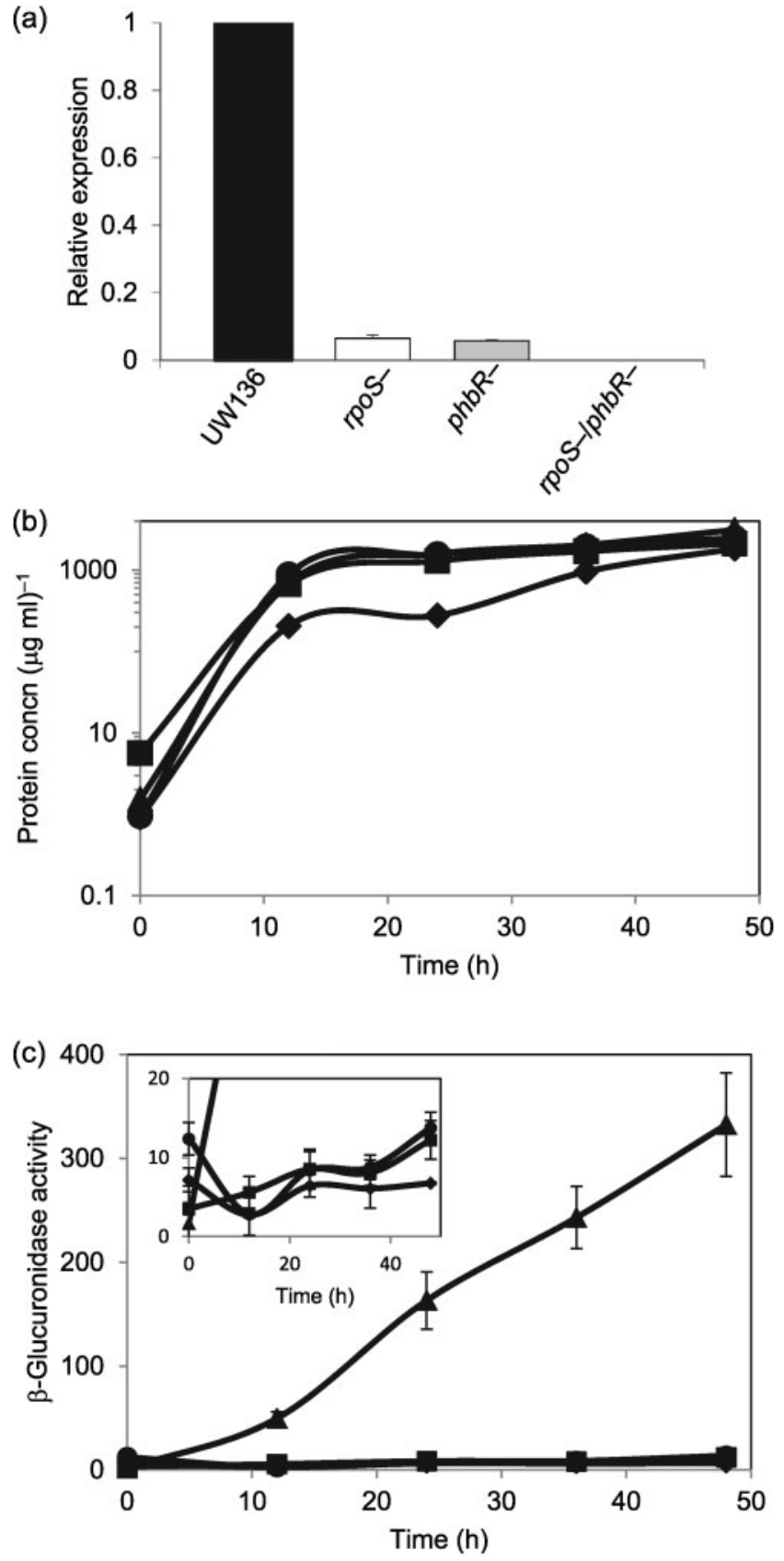

Fig. 3. PhbR and RpoS as positive regulators of phbB. (a) qRTPCR analysis of $p h b B$ gene expression in $p h b R, r p o S$ and rpoSphbR mutants. The data are presented as fold changes of mRNA levels of rpoS (white bar), phbR (grey bar) and rpoS-phbR mutant strains relative to that of the wild-type UW136 (black bar). (b) Growth of strain AHW9 (A), and the isogenic phbR ( $\mathbf{\square}), r p o S(\mathbf{0})$ and $r p o S$-phbR $(\boldsymbol{)})$ strains. (c) $\beta$-Glucuronidase activity of a phbB-gusA gene fusion in strain AHW9 ( $\mathbf{A})$, and in the isogenic $\operatorname{phbR}(\boldsymbol{\square}), r p o S(\boldsymbol{\bullet})$ and rpoS-phbR $(\boldsymbol{\nabla})$ strains. A magnification of $\beta$-glucuronidase activity of mutant strains is shown in the inset. indicating the requirement for RpoS in the transcription of phbR.

The presence of sequences R5-R6, proposed to be PhbRbinding sites and located upstream of phbR (Fig. 5a), suggested that PhbR may also act as an autoregulator. We therefore determined the effect of $p h b R$ mutation on the levels of $p h b R$ mRNA, as determined by qRT-PCR. As shown in Fig. 4(a), the $p h b R$ transcript was reduced by about $80 \%$ in the absence of PhbR, suggesting that it also acts as an autoactivator. In the rpoS-phbR double mutant, no $p h b R$ transcript was detected, indicating that both regulatory proteins, $\mathrm{PhbR}$ and $\mathrm{RpoS}$, are required for $p h b R$ transcription.

The effect of RpoS and PhbR on expression of $p h b R$ was further evaluated using a $p h b R::$ gusA gene fusion. Strain AHW5, a UW136 derivative carrying the wild-type $p h b R$ gene and a phbR::gusA gene fusion integrated into the chromosome within the scrX locus, and strain AHW6, a derivative of AHW5 in which the wild-type phbR copy is interrupted with a Km-resistance cassette, were constructed as described in Methods. The $\beta$-glucuronidase activity was determined during growth on PY sucrose medium Fig. 4(b). As shown in Fig. 4(c), the $\beta$-glucuronidase activity was higher in the strain carrying the wild-type $p h b R$ copy, while in strain AHW6, the absence of $p h b R$ reduced the $\beta$ glucuronidase activity. This result further supports the contention that PhbR acts as a positive autoregulator.

The positive effect of RpoS on $p h b R$ expression was also confirmed by the reduction of the $\beta$-glucuronidase activity in the AHW7 strain, which carries the rpoS mutation (Fig. 4c). Additionally, the rpoS-phbR mutant strain (AHW8) showed an activity similar to that of the AHW7 strain, supporting the importance of RpoS and PhbR in phbR expression.

\section{Binding of PhbR to the phbB promoter regions}

Sequence analysis of the $p h b R-p h b B$ intergenic region revealed two almost identical 18 bp sequences, named R1 and R2, centred at positions -134 and -155 upstream of the ATG start codon, where R1 overlaps the -35 region of $\mathrm{p}_{\mathrm{B}} 1$. These sequences have previously been proposed to be PhbR-binding sites (Peralta-Gil et al., 2002). Other less conserved 18 bp sequences, designated R3, R4, R5 and R6, are present in this region. The R5 and R6 sequences overlap the $\mathrm{p}_{\mathrm{R}} 2$ promoter (Peralta-Gil et al., 2002; Fig. 5a). To determine whether PhbR binds to the $p h b B$ and $p h b R$ promoter regions, the $\mathrm{PhbR}$ protein was purified as described in Methods. A $405 \mathrm{bp}$ DNA fragment (F1) corresponding to the intergenic $p h b B-p h b R$ region, as well as fragments of 108 (F2), 147 (F3) and $133 \mathrm{bp}$ (F4) containing the R1-R2, R3-R4 and R5-R6 sites, respectively, were amplified by PCR and subjected to an electrophoretic mobility shift assay (EMSA) with purified PhbR. Fig. 5(b, c) shows the binding of PhbR to the $405 \mathrm{nt}$ fragment spanning the $p h b R-p h b B$ intergenic region, as 

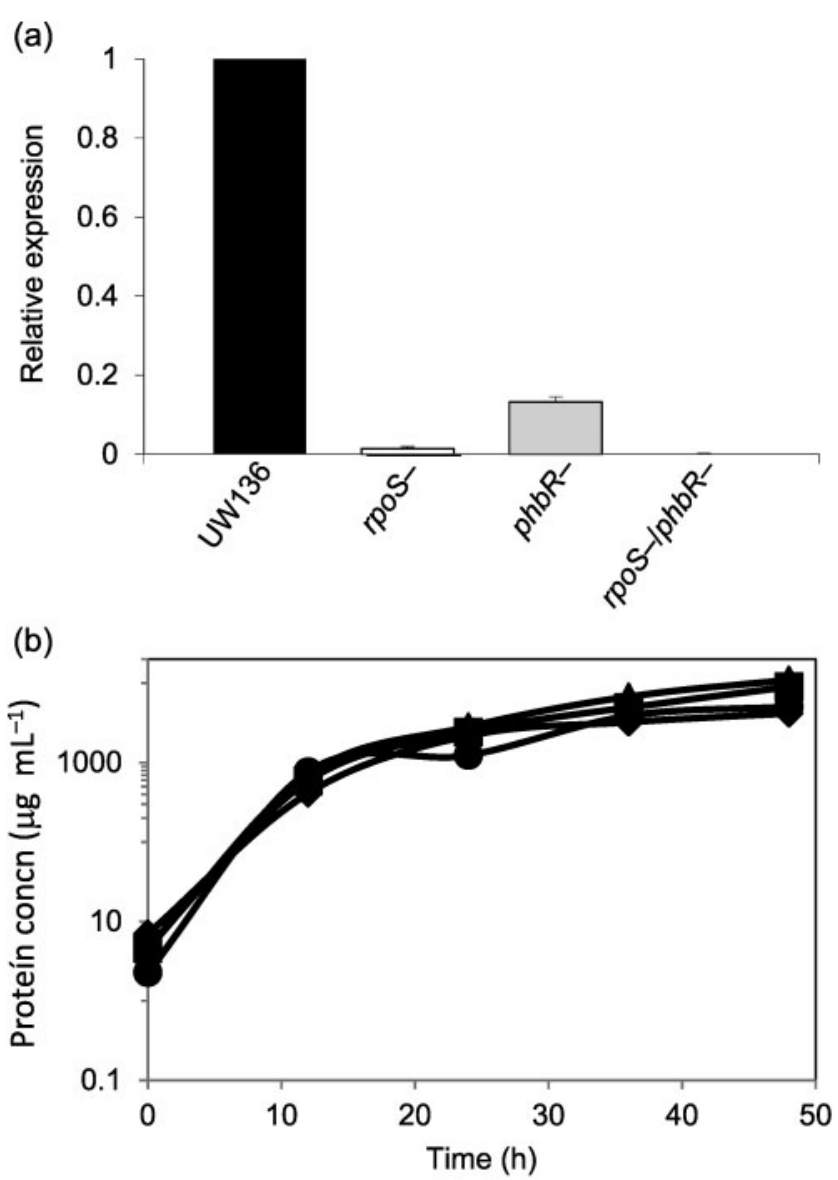

(c)

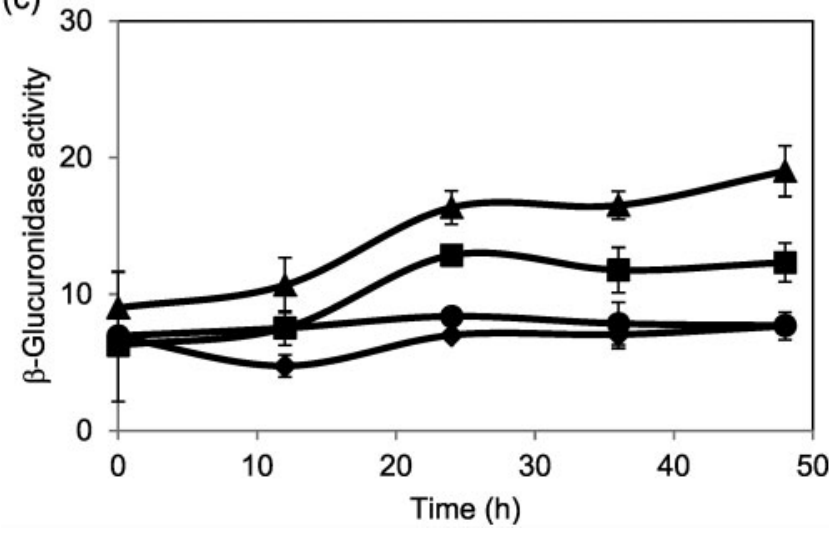

Fig. 4. $\mathrm{PhbR}$ and $\mathrm{RpoS}$ as positive regulators of $p h b R$. (a) qRTPCR analysis of phbR gene expression in the phbR and rpoS mutants. The data are presented as fold changes of mRNA levels of $r p o S$ (white bar), phbR (grey bar) and rpoS-phbR mutant strains relative to that of strain AHW5 (black bar). (b) Growth of wild-type UW136 ( $\mathbf{\Delta})$, and of the isogenic phbR $(\boldsymbol{\square}), \operatorname{rpoS}(\boldsymbol{\bullet})$ and rpoSphbR ( strains. (c) $\beta$-Glucuronidase activity of a phbB-gus $A$

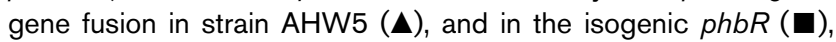
rpoS (৩) and rpoS-phbR ( $)$ strains. well as to the R1-R2-containing fragment F2. Binding of PhbR to the fragments containing the R1-R2 sites was observed at a PhbR concentration of $1.0 \mu \mathrm{M}$ (Fig. 5b, c). Some binding of PhbR to the larger fragment F1 was observed at a lower PhbR concentration of $0.2 \mu \mathrm{M}$ (Fig. $5 b)$. No binding of PhbR to fragments containing R3-R4 and R5-R6 sites was detected (data not shown), nor to non-specific psrA DNA (RRpsrA) used as a negative control. These results indicate that PhbR activates expression of $p h b B$ by binding specifically to the promoter region containing the $\mathrm{R} 1$ and $\mathrm{R} 2$ sequences.

\section{In vitro footprinting analysis}

DNase footprinting was performed to determine specific binding of $\mathrm{PhbR}$ to the direct repeated sequences TGTCACCAA- $\mathrm{N}_{4}$-CACTA and TGTCACCAA- $\mathrm{N}_{4}$-CAGTA (R1 and R2 sites) present in the -35 to -70 bp region upstream of the $\mathrm{p}_{\mathrm{B}} 1$ transcriptional start site for $p h b B$ (Fig. 6a). As shown in Fig. 6(b), PhbR protected the R1-R2 box at $0.5 \mu \mathrm{M}$, corroborating the data generated by EMSA and indicating that PhbR binds specifically within the R1-R2 boxes. Sequences located upstream and downstream of the PhbR-binding boxes R1-R2 were not protected, even at higher PhbR concentrations $(1.0-4.0 \mu \mathrm{M})$.

\section{DISCUSSION}

In general, negative regulators bind to promoters, interfering directly with RNA polymerase. In contrast, positive regulators bind to the upstream regions of the promoter, helping to recruit the polymerase and to start transcription (Browning \& Busby, 2004).

PhbR belongs to the AraC family of transcriptional activators, some of which bind 18-20 nt repeated sequences. We show here that PhbR binds specifically to the 18 bp R1 and $\mathrm{R} 2$ sites, where $\mathrm{R} 1$ overlaps the -35 region of the $\mathrm{p}_{\mathrm{B}} 1$ promoter (Fig. 6a). The position of the A. vinelandii R1 and R2 sites is similar to the position of some of the binding sites for MelR in the melAB promoter of E. coli. MelR binds four target sites, named $1^{\prime}, 1,2^{\prime}$ and 2, where site $2^{\prime}$ also overlaps the -35 region (Belyaeva et al., 2000). MelR binding to the less conserved site $\left(2^{\prime}\right)$ is weak and occurs only in the presence of melibiose (Belyaeva et al., 2000). Modification of the base sequence of this site removes the requirement for melibiose for MelR binding (Tamai et al., 2000). In A. vinelandii, the $\mathrm{R} 1$ site is almost identical to the R2 site, suggesting that no inducer is required for activation of $p h b B$ by PhbR.

The data presented in this study, together with previously published data (Peralta-Gil et al., 2002), confirm that PhbR activates the $p h b B$ gene.

In E. coli, the melR gene is expressed from a divergent promoter upstream of the melAB promoter. MelR also binds to a site that overlaps the -10 region of the melR 
(a)

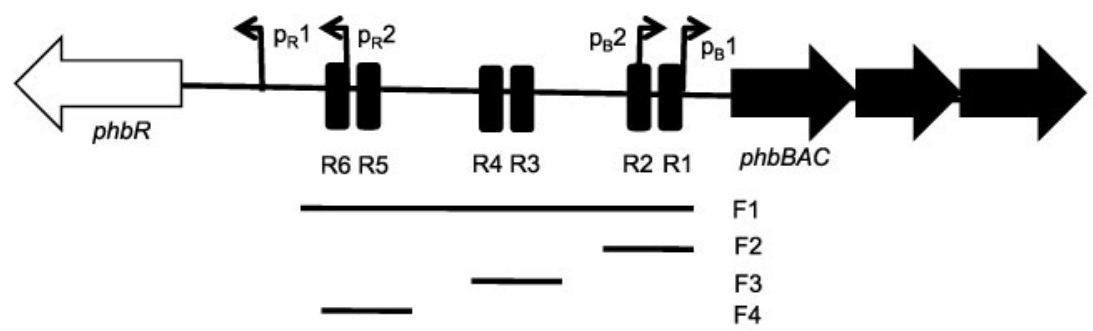

(b)
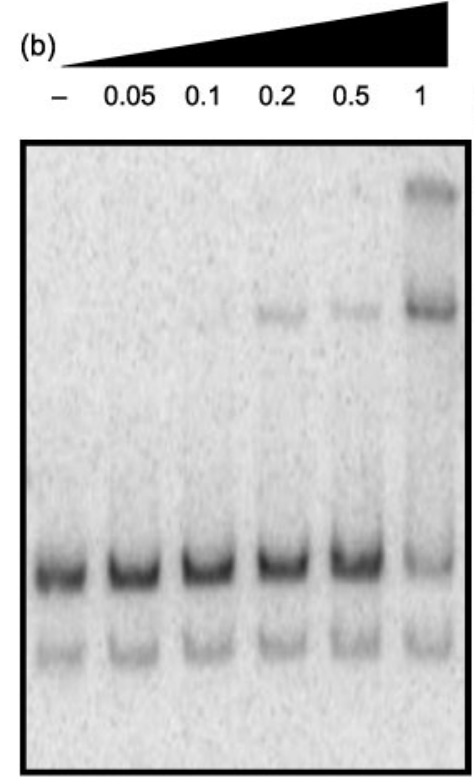

MBP-PhbR $(\mu \mathrm{M})$

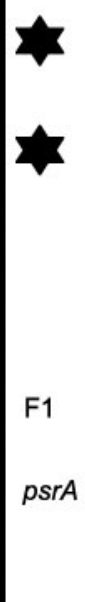

(d)

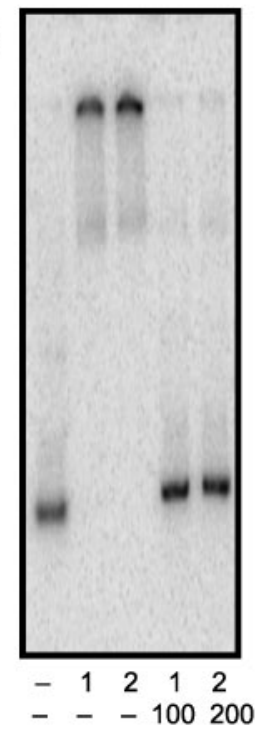

(c)
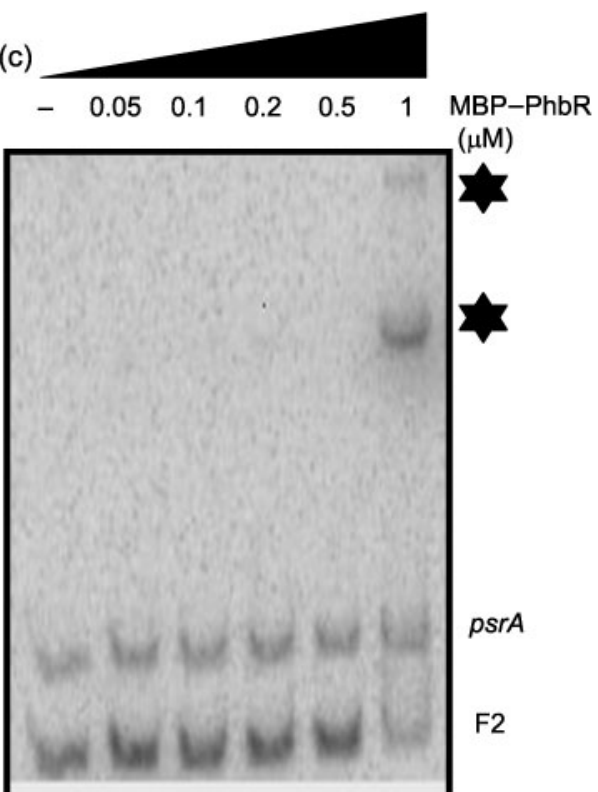

(e)

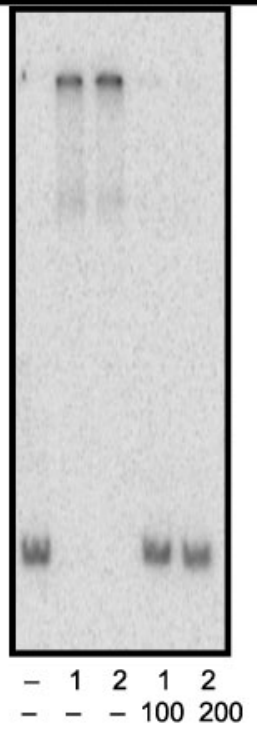

$\operatorname{PhbR}(\mu \mathrm{M})$ Fold excess cold DNA

Fig. 5. PhbR protein binds the $p h b B$ promoter region. (a) Schematic representation of the phbR-phbB intergenic region, showing the position of the $p h b B p_{\mathrm{B}} 1$ and $\mathrm{p}_{\mathrm{B}} 2$ and $p h b R \mathrm{p}_{\mathrm{R}} 1$ and $\mathrm{p}_{\mathrm{R}} 2$ promoters (transcription start sites) and the proposed PhbR-binding sites R1 to R6 (Peralta-Gil et al., 2002). The PCR F fragments used for the EMSA for the binding of PhbR are depicted. (b, c) EMSA showing the binding of various concentrations of PhbR $(0.05-1 \mu \mathrm{M})$ to the F1 fragment (b) or to the F2 fragment (c). Stars show PhbR-DNA complexes. (d) Competition assay for PhhR-F1 complex formation to verify the binding specificity. Competition reactions used specific unlabelled competitor ( $F 1$ fragment) at the concentrations shown. (e) Competition assay for PhhR-F2 complex formation to verify the binding specificity. Competition reactions used specific unlabelled competitor (F2 fragment) at the concentrations shown. 
(a)

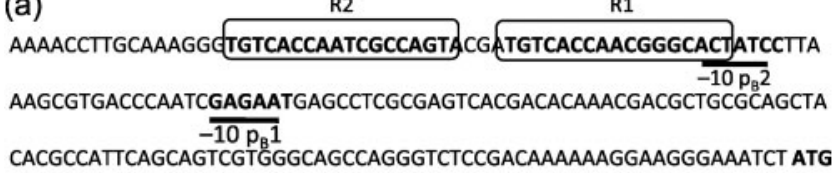

(b)

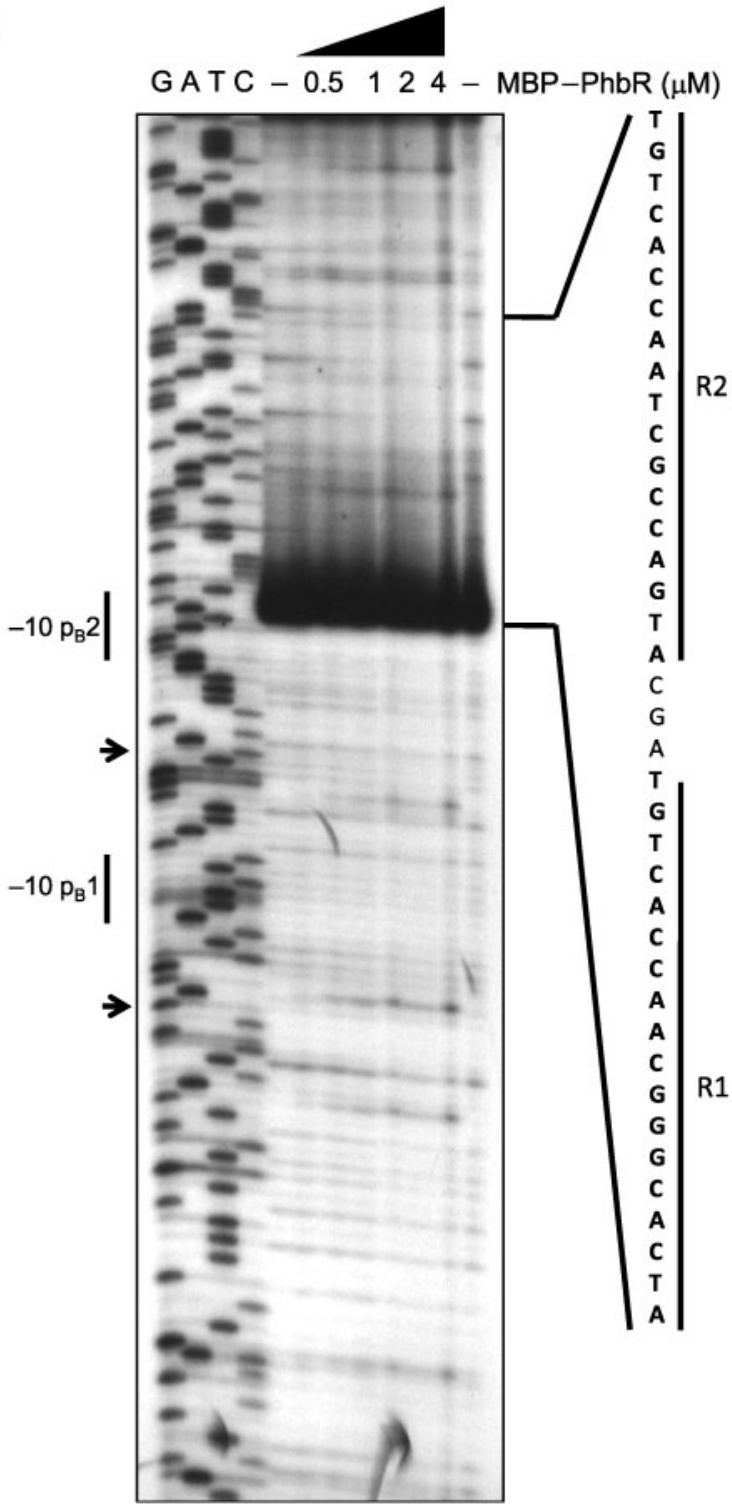

Fig. 6. (a) The phbB promoter region. The R1 and R2 sequences are boxed. The -10 regions for $p_{B} 1$ and $p_{B} 2$ are underlined. (b) DNase I footprinting analysis of the interaction of PhbR with the phbB promoter region. The reaction mixture was treated as described in Methods, and the sequencing ladder was generated with plasmid pCT2P and oligonucleotide footphbR4. -, Absence of $\mathrm{PhbR}$. The -10 regions of the $p_{B} 1$ and $p_{B} 2$ promoters are indicated. The arrows show the start sites for $p h b B$ transcription. The R1 and R2 sequences protected from degradation are also shown. promoter to downregulate its own expression (Wade et al., 2000; Samarasinghe et al., 2008).

The results presented here indicate that PhbR autoregulates its own expression positively. This regulation may be carried out in a direct manner, by binding to the R5 and R6 sites present upstream of the $\mathrm{p}_{\mathrm{R}} 1$ promoter region. However, no specific binding of PhbR to a fragment carrying R5 and R6 sites was observed in vitro in the EMSA assays. This result indicates either that PhbR indirectly activates its own transcription, or that a metabolite is required for PhbR binding to these less conserved R5 and R6 sites, similar to the requirement for melibiose in the binding of MelR to the less conserved site 2 (Belyaeva et al., 2000).

PhbR orthologues are present in Azotobacter strain FA8 (Pettinari et al., 2001) and in Pseudomonas sp. strain 61-3 (Matsusaki et al., 1998). In these bacteria, direct repeat sequences of 18 bp similar to the R1 and R2 PhbR-binding sites are present upstream of the PHB biosynthetic genes, which are also organized in a phbBAC cluster. Thus, in these bacteria, activation of the PHB biosynthetic operon by $\mathrm{PhbR}$ is expected to occur as in A. vinelandii.

Similar to PhbR, RpoS plays an important role in the activation of transcription of the $p h b B$ and $p h b R$ genes, as indicated by the negative effect of the $r p o S$ mutation on the levels of $p h b B$ and $p h b R$ mRNAs and the kinetics of transcription of $p h b B:: g u s A$ and $p h b R:: g u s A$ gene fusions, showing that induction of transcription occurs upon entrance into the stationary phase in an RpoS-dependent manner.

These observations are in agreement with previously published data that show a significant reduction of the phbR transcript levels initiated from the $\mathrm{p}_{\mathrm{R}} 2$ promoter in the rpoS mutant (Peralta-Gil et al., 2002). Whether RpoS directly activates $p h b R$ remains to be investigated. No RpoSrecognized sequences are present in the -10 sequences of the $\mathrm{p}_{\mathrm{R}} 1$ or $\mathrm{p}_{\mathrm{R}} 2$ promoters (Peralta-Gil et al., 2002), suggesting that RpoS control of $p h b R$ expression is indirect.

The control of PHB synthesis by RpoS is also in agreement with previous observations that indicate that in $A$. vinelandii, accumulation of rpoS mRNA, as well as $\mathrm{PHB}$, occurs mainly in cells entering the stationary phase and during the stationary phase (Castañeda et al., 2001; Sandercock \& Page, 2008; Martínez et al., 1997).

We propose that the activation of phbR expression by RpoS allows an increase in the concentration of PhbR in the stationary phase, which in turn further increases its concentration by autoactivation (in the presence of an inducer), allowing activation of the phbBAC biosynthetic operon and the synthesis of PHB in the stationary phase.

\section{ACKNOWLEDGEMENTS}

A. H.-E. thanks CONACYT for financial support during his $\mathrm{PhD}$ studies. M. C. thanks DGAPA for a postdoctoral fellowship. We thank Daniel Segura for critical reading of the manuscript. 


\section{REFERENCES}

Alexeyev, M. F., Shokolenko, I. N. \& Croughan, T. P. (1995). Improved antibiotic-resistance gene cassettes and omega elements for Escherichia coli vector construction and in vitro deletion/insertion mutagenesis. Gene 160, 63-67.

Bali, A., Blanco, G., Hill, S. \& Kennedy, C. (1992). Excretion of ammonium by a nifL mutant of Azotobacter vinelandii fixing nitrogen. Appl Environ Microbiol 58, 1711-1718.

Belyaeva, T. A., Wade, J. T., Webster, C. L., Howard, V. J., Thomas, M. S., Hyde, E. I. \& Busby, S. J. (2000). Transcription activation at the Escherichia coli melAB promoter: the role of MelR and the cyclic AMP receptor protein. Mol Microbiol 36, 211-222.

Browning, D. F. \& Busby, S. J. (2004). The regulation of bacterial transcription initiation. Nat Rev Microbiol 2, 57-65.

Castañeda, M., Sánchez, J., Moreno, S., Núñez, C. \& Espin, G. (2001). The global regulators GacA and $\sigma^{\mathrm{S}}$ form part of a cascade that controls alginate production in Azotobacter vinelandii. J Bacteriol 183, 6787-6793.

Cocotl-Yañez, M., Sampieri, A., Moreno, S., Núñez, C., Castañeda, M., Segura, D. \& Espin, G. (2011). Roles of RpoS and PsrA in cyst formation and alkylresorcinol synthesis in Azotobacter vinelandii. Microbiology 157, 1685-1693.

Hanahan, D. (1983). Studies on transformation of Escherichia coli with plasmids. J Mol Biol 166, 557-580.

Johnson, D. C., Unciuleac, M. C. \& Dean, D. R. (2006). Controlled expression and functional analysis of iron-sulfur cluster biosynthetic components within Azotobacter vinelandii. J Bacteriol 188, 7551-7561.

Kennedy, C., Gamal, R., Humphrey, R., Ramos, J., Brigle, K. \& Dean, D. (1986). The nifH, nifM, and nif $N$ genes of Azotobacter vinelandii: characterization by Tn 5 mutagenesis and isolation from pLARF1 gene banks. Mol Gen Genet 205, 318-325.

Lowry, O. H., Rosebrough, N. J., Farr, A. L. \& Randall, R. J. (1951). Protein measurement with the Folin phenol reagent. J Biol Chem 193, 265-275.

Martínez, P., Guzman, J. \& Espín, G. (1997). A mutation impairing alginate production increased accumulation of poly- $\beta$-hydroxybutyrate in Azotobacter vinelandii. Biotechnol Lett 19, 909-912.

Matsusaki, H., Manji, S., Taguchi, K., Kato, M., Fukui, T. \& Doi, Y. (1998). Cloning and molecular analysis of the poly(3-hydroxybutyrate) and poly(3-hydroxybutyrate-co-3-hydroxyalkanoate) biosynthesis genes in Pseudomonas sp. strain 61-3. J Bacteriol 180, 6459-6467.

Navarro Llorens, J. M., Tormo, A. \& Martínez-García, E. (2010). Stationary phase in Gram-negative bacteria. FEMS Microbiol Rev 34, $476-495$.
Noguez, R., Segura, D., Moreno, S., Hernandez, A., Juárez, K. \& Espin, G. (2008). Enzyme I Itr, NPr and IIA ${ }^{\mathrm{Ntr}}$ are involved in regulation of the poly- $\beta$-hydroxybutyrate biosynthetic genes in Azotobacter vinelandii. J Mol Microbiol Biotechnol 15, 244-254.

Page, W. J. \& von Tigerstrom, M. (1978). Induction of transformation competence in Azotobacter vinelandii iron-limited cultures. Can J Microbiol 24, 1590-1594.

Peralta-Gil, M., Segura, D., Guzmán, J., Servín-González, L. \& Espín, G. (2002). Expression of the Azotobacter vinelandii poly- $\beta$-hydroxybutyrate biosynthetic phbBAC operon is driven by two overlapping promoters and is dependent on the transcriptional activator PhbR. J Bacteriol 184, 5672-5677.

Pettinari, M. J., Vázquez, G. J., Silberschmidt, D., Rehm, B., Steinbüchel, A. \& Méndez, B. S. (2001). Poly(3-hydroxybutyrate) synthesis genes in Azotobacter sp. strain FA8. Appl Environ Microbiol 67, 5331-5334.

Samarasinghe, S., El-Robh, M. S., Grainger, D. C., Zhang, W., Soultanas, P. \& Busby, S. J. (2008). Autoregulation of the Escherichia coli melR promoter: repression involves four molecules of MelR. Nucleic Acids Res 36, 2667-2676.

Sambrook, J., Fritsch, E. E. \& Maniatis, T. (1989). Molecular Cloning: a Laboratory Manual, 2nd edn. Cold Spring Harbor, NY: Cold Spring Harbor Laboratory.

Sandercock, J. R. \& Page, W. J. (2008). RpoS expression and the general stress response in Azotobacter vinelandii during carbon and nitrogen diauxic shifts. J Bacteriol 190, 946-953.

Segura, D. \& Espín, G. (1998). Mutational inactivation of a gene homologous to Escherichia coli $p t s P$ affects poly- $\beta$-hydroxybutyrate accumulation and nitrogen fixation in Azotobacter vinelandii. J Bacteriol 180, 4790-4798.

Segura, D., Cruz, T. \& Espin, G. (2003). Encystment and alkylresorcinol production by Azotobacter vinelandii strains impaired in poly- $\beta$-hydroxybutyrate synthesis. Arch Microbiol 179, 437443.

Tamai, E., Belyaeva, T. A., Busby, S. J. W. \& Tsuchiya, T. (2000). Mutations that increase the activity of the promoter of the Escherichia coli melibiose operon improve the binding of MelR, a transcription activator triggered by melibiose. J Biol Chem 275, 17058-17063.

Wade, J. T., Belyaeva, T. A., Hyde, E. I. \& Busby, S. J. W. (2000). Repression of the Escherichia coli melR promoter by MelR: evidence that efficient repression requires the formation of a repression loop. Mol Microbiol 36, 223-229.

Edited by: M. F. Hynes 